\title{
Persistence of Human Immunodeficiency Virus and Vaccine
}

\section{Development}

\section{Naoki Yamamoto* \\ Department of Molecular Virology, Tokyo Medical and Dental University, Japan}

*Corresponding author: Naoki Yamamoto, National Institute of Infectious Diseases,

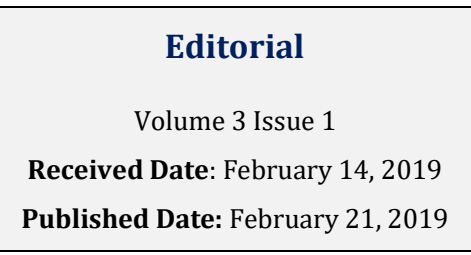

Department of Molecular Virology, Tokyo Medical and Dental University, Tokyo, Japan, Email:

\section{Introduction}

Highly active anti-retroviral therapy (HAART) capable of suppressing the proliferation of HIV has been developed and this effective therapy for HIV infection has become widely available. Thanks to this, it seems now that the former fatal illness has changed to be immortal. In addition, PrEP (Preexposure prophylaxis) that can effectively prevent HIV infection using anti-HIV drugs has become a hot topic, recently. This pharmacological regimen is used prior to sexual activity in a group at a risk of infection, and the best known is the combination regimen of two reverse transcriptase inhibitors, Truvada [1]. According to a recent study, PrEP is routinely used for two and a half years, effectively preventing HIV infection [2]. Needless to mention, it is not to protect against sexually transmitted diseases and infectious diseases other than HIV. There seems to be a false idea that using a PrEP no longer requires a condom. Of course, although it does not deny the importance of the PrEP and its dissemination, it is apparent that education which does not elicit misunderstanding is also necessary. Otherwise, it could prevent HIV infection but can even help increase other sexually transmitted diseases. In other words, it is recommended that PrEP be used in combination with safer sex practices.

\section{Basic Research on AIDS/HIV Is Still A Key for Solution}

There is even a tendency that fundamental research on AIDS and HIV is no longer necessary because of the success of treatment of HIV infection using antiviral drugs, but this is clearly a wrong idea. According to the UNAIDS Fact sheet, about 70 million people have already experienced HIV since the 1980s. About 30 million people are living with HIV and as many as 37 million people around the world suffer from HIV around 2016 [3]. Even now, there are 1.8 million people infected with HIV newly every year worldwide, yet we have no measures for prevention of infection. For example, what do you answer to the question "do you want your child to receive PrEP?". Probably, most answers will be "no". Although this is powerful, it is not a usual medication therapy for the general public. We do not even know an effective way to eliminate viruses from already infected people. An infected person must keep taking drugs. That is why we must focus on the basic research on HIV now. Otherwise, we cannot expect further clinical progress such as developing truly effective HIV vaccines or eliminating the virus from infected people.

\section{Prevention of HIV Infection}

Like all other infections, prevention of infection by the vaccine was the final goal of AIDS/HIV research since the discovery of AIDS and HIV. An examination of HIV vaccine candidate (HVTN 702) in South Africa is ongoing [4]. This study is based on the so-called RV 144 test which was conducted in Thailand before [5]. The vaccine used there, which had some effect, reduced the risk of HIV by approximately $30 \%$. This result was considered sufficient to suggest that the RV144 vaccine may give better results. Therefore, under the initiative of NIH, a large-scale test is being conducted targeting the HIV subtypes prevailing in South Africa. Even though these vaccine candidates generally show promising safety and immunogenicity profiles, clinical trials do not yet seem to prove that the vaccine can prevent HIV infection. 


\section{Journal of Infectious Diseases \& Travel Medicine}

In developing the HIV vaccine, it is important to think back to the starting point once how the viral infection is established in the victims and whether vaccine development is really possible against this unique virus. Of course, everyone wants to realize an effective HIV vaccine, but expectation and whether it is scientifically possible is different. The trial of vaccine production against HIV seems to be a level of difficulty that none of the researchers have ever experienced. Needless to say, all these difficulties come from the unique nature of this virus, and is a very challenging issue. Below I would like to describe some of those that make HIV vaccine development particularly difficult.

The vast majority of vaccines that have been put into practical use so far target the pathogens that exhibit little genetic variation and relatively simple viruses. Indeed, all the vaccines that have been used in the past are for infectious diseases such as Japanese encephalitis, rabies, hepatitis $\mathrm{A}$, hepatitis $\mathrm{B}$, polio, measles, rubella, mumps, varicella, yellow fever, rota, and HPV infection. From that point of view, HIVs' ability to mutate easily is undoubtedly makes this virus a tremendously difficult target and it will be a big wall in developing effective vaccine. It is theoretically impossible to use live attenuated vaccines against viruses that are so easily mutated and thus forming large quasispecies. Similarly, inactivated vaccines strategy is useless since we do not know how many or which mutant populations are to be targeted. How to deal with HIV quasispecies is also a tremendous issue to be studied since this suggests that we need to find antigens that can respond to all kinds of invading HIV with just a few vaccinations. We cannot use knowledge obtained in developing attenuated live vaccine, inactivated vaccine and subunit vaccine, all of which are big heritages acquired by humanity so far. If so, question may be arisen why influenza is being prevented by vaccine though influenza is also quite variable in the same way. However, in case of influenza, viral strains that are prevalent each year are predictable to a certain extent and they are special type of vaccines that require conditioning of the vaccine each year. Therefore, the circumstances are totally different from those in the case of HIV. Of course, even in development of effective influenza vaccines, its difficulty will be much higher in level when compared to other vaccines.

\section{Viral Invasion into the Body}

In vaccine development, it is necessary to inquire exact nature of the targeted virus, especially as to its invasion process in the body leading to viral proliferation and disease onset. The more we learn about HIV, the more we know that it is more unique compared to the viruses to which vaccine strategy is already available. An Australian virologist, F. Fenner, made great contributions to our understanding of viral invasion into the living body, its proliferation and disease onset through his research on the ectromelia [6]. Ectromelia virus is a mouse poxvirus and invades from the skin, proliferates in the invading locus, and its viral titer reaches nearly the peak before infection in the lesion becomes macroscopically apparent. And between the infection and the end of the incubation period, the virus travels from the skin to the local lymph nodes where it amplifies. A small amount of virus enters the bloodstream (primary viremia) and undergoes phagocytosis by cells of the reticuloendothelial system. The virus grows in these target cell-rich organs (e.g. liver and spleen), causing secondary viraemia with necrosis of infected cells adjacent to the sinusoid. Viruses distributed in this manner and cause local infection of cells in the epidermis, the final target tissue. And the clinical recovery and the disappearance of the virus will closely interlock with the appearance of neutralizing antibody. This interpretation of the virulence of mouse pox very well describes the phenomena occurring in smallpox, chicken pox, measles and so on.

\section{Early Events after HIV Infection}

Probably, one would say that the mouse pox could be a good laboratory model for the study of acute rash but not for HIV because it is too different from HIV and is not helpful. But I do not think so because the essence of virus infection is the same. Many studies have been done in the experimental system of SIV / SHIV as to the phenomena occurring early in the invasion of HIV and subsequent infection process. Several SIV / SHIV studies using macaque focus on infection of the cervical vagina by sexual intercourse because sexual contact, especially infection from men to women, is the biggest problem in AIDS. Hu et al. initially determined the minimal time that the SIV must remain contacting with the genital mucosa for SIV to move from the vaginal lumen into the mucosa. As a result, they found that SIV enters the vaginal mucosa within 60 minutes of vaginal exposure, predominantly infecting intraepithelial dendritic cells, and that SIVinfected cells are located in draining lymph nodes within 18 hours of vaginal SIV exposure [7]. As such, insights can be made about the anatomical sites and cell types at which the infection occurs and thus where the preventive approach should be targeted. The involvement of CCR5 + $\mathrm{CD} 4+\mathrm{T}$ cells in the lamina propria of the endometrium is also suggested as the earliest target cell of SIV infection $[8,9]$. Cells productively infected with viruses are detected at this site within 2 to 3 days of virus challenge and local lesions of infection can be detected immediately thereafter. From there, virus transmission occurs in the 


\section{Journal of Infectious Diseases \& Travel Medicine}

local lymph nodes and the virus spreads to secondary lymphoid tissues including gut-associated lymphoid tissues (GALT) $[10,11]$. The virus might initially infect antigen-presenting cells such as dendritic cells, macrophages and Langerhans cells in the vagina and then subsequent rounds of replication occur in other distant lymphoid tissues to form viral reservoirs [12]. Recent studies also suggest that target cells susceptible to HIV early after viral invasion may be widely distributed in female reproductive tracts than previously thought. Lesions infected with viruses have also been detected across various tissues of the female reproductive tract, including the ovaries, cervix and regional draining lymph nodes. If these observations apply to humans, vaccine prevention must target cells throughout the female reproductive tract.

Though the basics are similar, it is also true to say that the very difference between HIV and other viruses controllable by vaccines is the greatest hurdle for HIV vaccine development. HIV is unique because invasive infection into the initial target cells including dendritic cells, monocytic cells and CD4 T cells, is expected to occur very early upon infection. Roughly saying, other viruses such as ectromelia require a considerably long time and undergo several steps of virus proliferation in different cells and regions before they reach the final target cells. More importantly, these viruses cannot establish persistent infection eventually. In the meantime, the anamnestic response of the immunity acquired by the vaccine occurs and vaccines fulfills its purpose against these viruses. While in SIV/SHIV/HIV infection, critical process is extremely short posing a serious challenge to HIV vaccine development.

\section{What is Needed for Prophylactic Vaccines Against HIV}

It is also important to recognize the fact that in most vaccines available so far, virus infection actually occurs in the host while they are still enough to prevent an onset of the diseases. In other words, these vaccines can actually prevent the onset without preventing infection, in general. But this is not acceptable with a retrovirus vaccine. As described, HIV establishes persistent infection very quickly and HIV can be completely hidden from immune surveillance by incorporating its genome into the host cell's DNA. Once it happens, it is not possible to remove HIV, and CD4 T cells are gradually depleted by mutant viruses that appear newly by activation of host cells. Unfortunately, immunity acquired by the past infection is helpless.
All these facts strongly indicate that any HIV vaccine has very narrow window temporally to prevent viral infection. Therefore, HIV vaccines need to be extremely effective because the vaccine must thereby repel all attempts by viruses adhering to target cells in order to establish infection in the host. Though persistent, point is whether the humoral and cellular immunity acquired by the vaccination can completely block viral integration into the host cell genome and resulting persistent infection within this short time.

Since HIV primarily infects cells via the blood and reproductive pathways, target cells are prepared in front of them as they invade. Again, the important point is that $\mathrm{HIV}$ can infect the final target cells such as CD4 T cells and monocyte cells, at very early stages directly, leading to the formation of the pool of latent HIV reservoirs. Among these short periods of time, will immune responses (humoral and cellular) conferred by the vaccine be able to suppress infection at the same time? HIV also provides a number of other challenges. Naturally, the vaccine is to give immunity to the host, and it is necessary to activate lymphocytes, especially $\mathrm{T}$ and $\mathrm{B}$ cells. But in the case of HIV, this is an extremely dangerous event. It is because the activation of CD4 $\mathrm{T}$ cells will provide a suitable growth environment for HIV proliferation there. Therefore, in the development of HIV vaccines, it is necessary to develop a vaccine that does not activate $\mathrm{CD} 4 \mathrm{~T}$ lymphocytes excessively, contradicting conventional common sense. This also represents a difficulty in developing HIV vaccines. In addition, HIV has unique genes such as tat and if that are known to interfere with the induction of potent anti-HIV reactions in infected as well as uninfected cells.

Finally, it is often said that "even with a partially effective vaccine, the burden of HIV infection will be significantly reduced over time". It is undoubtedly true from epidemiological point of view. However, what I am seeking as a virologist, is more effective vaccine that can protect the majority of vaccinees from HIV infection and onset. Such vaccine will be achieved only with a very unique idea, a tremendous effort and fortune. No one has succeeded in developing vaccines against retroviruses, not just HIV, so far. HIV must be a formidable enemy.

\section{References}

1. Desai M, Field N, Grant R, McCormack S (2017) Recent advances in pre-exposure prophylaxis for HIV. BMJ 359: 5011. 
2. Garnett GP, Krishnaratne S, Harris KL, Hallett TB, Santos M, et al. (2017) Cost-Effectiveness of Interventions to Prevent HIV Acquisition.

3. (2018) Global HIV \& AIDS statistics.

4. (2016) HVTN702.

5. Rerks-Ngarm S, Pitisuttithum P, Nitayapan S, Kaewkungwal J, Chiu J, et al. (2009) Vaccination with ALVAC and AIDSVAX to prevent HIV-1 infection in Thailand. N Engl J Med 361(23): 2209-2220.

6. Fenner F (2016) Classic paper: Fenner on the exanthemata. Rev Med Virol 16(6): 353-363.

7. $\mathrm{Hu} \mathrm{J}$, Gardner MB, Miller CJ (2000) Simian immunodeficiency virus rapidly penetrates the cervicovaginal mucosa after intravaginal inoculation and infects intraepithelial dendritic cells. J Virol 74(13): 6087-6095.

8. Sugimoto C, Nakamura S, Hagen SI, TsunetsuguYokota Y, Villinger F, et al. (2012) Glycosylation of simian immunodeficiency virus influences immunetissue targeting during primary infection, leading to immunodeficiency or viral control. J Virol 86(17): 9323-9336.

9. Ma ZM, Dutra J, Fritts L, Miller CJ (2016) Lymphatic Dissemination of Simian Immunodeficiency Virus after Penile Inoculation. J Virol 90(8): 4093-4104.

10. Heise C, Miller CJ, Lackner A, Dandekar S (1994) Primary acute simian immunodeficiency virus infection of intestinal lymphoid tissue is associated with gastrointestinal dysfunction. J Infect Dis 169(5): 1116-1120.

11. Veazey RS, DeMaria M, Chalifoux LV, Shvetz DE, Pauley DR, et al. (1998) Gastrointestinal tract as a major site of $\mathrm{CD} 4+\mathrm{T}$ cell depletion and viral replication in SIV infection. Science 280(5362): 427431.

12. Miller CJ (1998) Localization of Simian immunodeficiency virus-infected cells in the genital tract of male and female Rhesus macaques. J Reprod Immunol 41(1-2): 331-339. 